\title{
Psychological Well-being and Social Supports among the Rural Left-Behind Elderly in Ethnic Minority Areas
}

\author{
Jian $\mathrm{HU}^{1, \mathrm{a}}, \mathrm{Si}-Y$ Yuan $\mathrm{LI}^{1, \mathrm{~b}}$ \\ ${ }^{1}$ School of Public Management, Guizhou University of Finance and Economics, Guiyang, China \\ ajianhucdc@126.com, ${ }^{b} 786444880 @ q q . c o m$
}

Keywords: Left-behind old people, Psychological well-being, Social support.

\begin{abstract}
To describe the status of psychological well-being, and analyze the relationship between the psychological well-being and the social supports among the rural left-behind elderly in ethnic minority areas. [Methods] A cross-sectional survey was conducted with a structured questionnaire among 249 old people at the age of 60 or older in August 2013 in the eight villages of Zhuchangping town in Xingyi city, Qianxinan Buyei and Miao autonomous prefecture of Guizhou province, China. [Results] The total score of psychological well-being was (26.41 \pm 4.22$)$, and the score of adaptation dimension was $(6.10 \pm 2.06)$ among the left-behind elderly, which were lower than the scores among the non left-behind elderly $(P<0.05)$. The score of personality, emotion, interpersonal relation and cognition dimensions among the left-behind was $(4.18 \pm 1.30),(4.57 \pm 1.38),(6.48 \pm 1.39)$, and $(5.07 \pm 1.67)$, respectively. There was a significant difference in the total score of psychological well-being among the old people with different characteristics of total social support, subjective support, support utilization level, subjective support outside family, visiting a doctor conveniently, mental health consultation, economic source, financial relief, and talking on phone with children every week $(P<0.05$ or $P<0.01)$. [Conclusion] The status of psychological well-being is worse among the rural left-behind elderly. The psychological well-being is related to the social supports.
\end{abstract}

\section{Introduction}

The rural left-behind elderly refers to the old people over sixty who still lived in the rural areas while their children works outside their county as migrant workers for six months or longer [1]. The problem of psychological well-being is serious among the rural left-behind elderly [2-3] and social supports may influence their psychological well-being [4]. This survey was conducted with a structured questionnaire among 147 rural left-behind old people and 102 non left-behind in Xingyi county, Qianxina Buyi and Miao autonomous prefecture of Guizhou province, China in August 2013. The aim of this study was to explore the status of psychological well-being among the rural left-behind elderly, examine the relationship between the psychological well-being and the social supports, and provide a scientific evidence for formulating and improving relevant policy for rural left-behind old people.

\section{Methods}

\section{Study Area and Population}

Zhuchangping town is a poverty-striven area with a higher representative in Xingyi county, including eight administrative villages of Zhuchangping, Tianwan, Yakouzhou, Baoshang, Zhangwan, Maocaowan, Yaxitian, and Longtan. About 249 old people aged 60 or older were interviewed in the eight administrative villages. There was the proportion of 59.04 percent for the left-behind elderly, of 40.96 percent for the non left-behind; of 45.8 percent for male, of 54.2 percent for female; of 43.4 percent at the age of $60-69$, of 44.2 percent at the age of 70-79, of 12.4 percent at the age of $\geq 80$. About 90 percent of the old people worked as farmers, 10 percent as non-farmers. 74.7 percent were married, and 25.3 percent did not. 98.8 percent reported ethnic Han, and 1.2 percent 
reported ethnic minorities. 15.3 percent of the old people were poor or low-income residents, and 84.7 percent did not.

\section{Data Collection and Measurement}

A one to one interview was performed with a structured questionnaire, which was designed according to the Questionnaire of Psychological Well-being for the Elderly by Zhenyun Wu in the Institute of Psychology, Chinese Academy of Sciences [5], and the Understanding Social Support Scale and Social Support Rating Scale in a Handbook of Psychological Health Assessment Scale [6]. With the coordination of Guizhou province center for disease control and prevention, the local township civil affairs department and township health center, the elderly were organized in the village offices or clinics by the village committees. The project members, the professional personnel of Guizhou province center for disease control and prevention, and the doctors of local township health centers were trained as investigators.

The study included three aspects. (1) Social demographic characteristics of the rural elderly. (2) The status and evaluation of social supports. The status of social supports included living style (living alone at home, living with children, living in gerocomium), economic source (themselves/their spouses, children/social relief), poor/low-income resident (yes, no), allowance of difficult life (yes, no), older people bonus (yes, no), medical insurance or New Rural Cooperative Medical System (yes, no), new rural endowment insurance (yes, no), talking on phone with children every week (yes, no), visiting a doctor conveniently (yes, no), mental health consultation (yes, no), old people recreation place (yes, no), old people compensative service (yes, no), old people cultural organization (yes, no), attending to old people recreation (yes, no). A binary variable "Yes" was assigned in "1", and "No" in " 0 " . An ordinal variable was assigned in "1", "2", "3", etc. The evaluation of total social supports included three dimensions of subjective support, objective support and support utilization level, with 12 items for each dimension. The subjective support referred to the support of the subjective experience. In other words, what extent of the emotional experience or satisfaction the old people felt? It was measured by the Understanding Social Support Scale [5]. The objective support referred to an objective, visible, or actual support, including a direct material aid and the existence and participation of social network and community relations. The support utilization level referred to the status of using social supports for old people. A 1-7 scoring method was applied for each item, namely, highly disagree, disagree, slightly disagree, neutral, somewhat agree, agree, and highly agree. The score of subjective support dimension was the cumulative score of each item or of the subjective support within family and the subjective support outside family. The scores of objective support and support utilization level were calculated by the scoring method of objective support and utilization level in the Social Support Rating Scale [6]. The score of total social support included the cumulative score of a subjective support, objective support and support utilization level. With an average score as a critical value, the total social support, subjective support (within family, outside family), objective support, and support utilization level were divided into two groups of "high" and "low". The "high" group was assigned in "1", and the "low" group in " 0 " [7]. (3) The status of psychological well-being was measured by 50 items in five dimensions of personality, emotion, adaptation, interpersonal relation and cognition in the Questionnaire of Psychological Well-being for the Elderly. There were 8, $9,12,12$, and 9 items in the personality, emotion, adaptation, interpersonal relation and cognition dimension, respectively. There were two groups of "yes" and "no" for each item. The "yes" group was assigned in " 1 ", and the "no" group in " 0 ". The total score of the psychological well-being was the sum of each dimension score. The higher the score, the better the psychological well-being. The full score of psychological well-being was 50. Cronbach coefficient was 0.71 for the psychological well-being, and 0.76 for the total social support, indicating a higher internal consistency reliability of the questionnaire.

\section{Data Analysis}

$t$ or $F$ test was performed using SPSS17.0. 


\section{Results}

\section{Psychological Well-being by the Left-behind Elderly}

There was a statistically significant difference in the total score of psychological well-being and adaptation dimension between the left-behind elderly and the non left-behind. See Table 1.

Table 1 psychological well-being by the left-behind elderly $(\bar{x} \pm \mathrm{s})$

\begin{tabular}{cccccccc}
\hline Left-behind elderly & $n$ & Total score & Personality & Emotion & Adaptation & Interpersonal relation & Cognition \\
& & & & & & & \\
\hline Yes & 147 & $26.41 \pm 4.22$ & $4.18 \pm 1.30$ & $4.57 \pm 1.38$ & $6.10 \pm 2.06$ & $6.48 \pm 1.39$ & $5.07 \pm 1.67$ \\
\hline No & 102 & $27.70 \pm 4.64$ & $4.15 \pm 1.41$ & $4.72 \pm 1.48$ & $6.75 \pm 1.96$ & $6.66 \pm 1.66$ & $5.43 \pm 1.48$ \\
\hline
\end{tabular}

Note: The total score of the psychological well-being $\mathrm{t}=5.16, \mathrm{P}=0.024$; Adaptation dimension $\mathrm{t}=6.09$, $\mathrm{P}=0.014$.

\section{Psychological Well-being by Social Support}

There was a significant difference in the total score of psychological well-being among the old people with different characteristics of status and evaluation of social supports, e.g. total social support, subjective support, support utilization level, subjective support outside family, visiting a doctor conveniently, mental health consultation, economic source, allowance of difficult life, and talking on phone with children every week. See Table 2.

Table 2 psychological well-being by social support

\begin{tabular}{|c|c|c|c|c|c|}
\hline Variable & $n$ & $x$ & $s$ & $t$ & $P$ \\
\hline Total social support & & & & 1.97 & 0.050 \\
\hline High & 135 & 27.44 & 4.66 & & \\
\hline Low & 114 & 26.34 & 4.09 & & \\
\hline Subjective support & & & & -2.44 & 0.016 \\
\hline High & 135 & 27.55 & 4.79 & & \\
\hline Low & 114 & 26.21 & 3.87 & & \\
\hline Support utilization level & & & & -2.88 & 0.004 \\
\hline High & 128 & 27.71 & 4.32 & & \\
\hline Low & 121 & 26.11 & 4.43 & & \\
\hline Subjective support outside family & & & & -3.52 & 0.001 \\
\hline High & 142 & 27.77 & 4.55 & & \\
\hline Low & 107 & 25.82 & 4.05 & & \\
\hline Visiting a doctor conveniently & & & & -6.74 & 0.000 \\
\hline Yes & 180 & 27.89 & 4.48 & & \\
\hline No & 69 & 24.44 & 3.20 & & \\
\hline \multicolumn{6}{|l|}{ Mental health consultation } \\
\hline Yes & 8 & 31.13 & 6.03 & -2.75 & 0.006 \\
\hline No & 241 & 26.80 & 4.32 & & \\
\hline Economic source & & & & 2.22 & 0.027 \\
\hline Themselves or spouses & 173 & 27.35 & 4.34 & & \\
\hline Children or social relief & 76 & 26.00 & 4.54 & & \\
\hline Allowance of difficult life & & & & 2.93 & 0.004 \\
\hline Yes & 5 & 32.60 & 4.62 & & \\
\hline No & 244 & 26.82 & 4.37 & & \\
\hline Talking on phone with children & & & & -3.29 & 0.001 \\
\hline Yes & 145 & 27.70 & 4.37 & & \\
\hline No & 104 & 25.87 & 4.32 & & \\
\hline
\end{tabular}

\section{Discussion}

The results showed that the total score of psychological well-being and the score of adaptation dimension were lower for the rural left-behind elderly than for the rural non left-behind $(P<0.05)$, 
which was consistent with the results of other research [8]. This suggests that the status of psychological well-being was worse, and the capability of coping with stress events was weaker for the rural left-behind elderly, which should be taken into consideration seriously. There was a significant difference in the total score of the psychological well-being with different total social support, subjective support and subjective support outside family $(P<0.05$ or $P<0.01)$. The study showed that the rural left-behind elderly had not been treated preferentially in the New Rural Cooperative Medical System and New Rural Endowment Insurance System. The rural left-behind elderly reported that they did not get enough respects and supports in the local society. Social supports are the spiritual and material supports of the individuals receiving from their own social relations, which have buffer effects for stress events, and the main effects (namely, generally gain functions) for maintaining general good emotional experiences [7]. This indicates that social support environments including the relevant policy environments might be improved for the rural left-behind elderly to increase their satisfaction degree of emotional experiences. In addition, the results showed that there was a significant difference in the total score of the psychological well-being with different economic source, allowance of difficult life and talking on phone with children every week $(P<0.05$ or $P<0.01)$. The survey showed that the rural left-behind elderly had few financial supports from their children, but offered a lot of financial supports to their children. This indicates that the children might be encouraged to increase financial supports to their rural left-behind parents and to communicate often with them on telephone. Relevant departments might strengthen the social assistance for the rural left-behind elderly. Moreover, there was a significant difference in the total score of psychological well-being with different characteristics of visiting a doctor conveniently and mental health consultation $(P<0.05$ or $P<0.01)$. The local township health centers established the elderly health archives in the primary public health service program, and carried out the screening examination of mental health diseases for the elderly. This indicates that the policy might be formulated to encourage the local health personnel to provide the mental health counseling further for the left-behind elderly in the rural communities. Finally, there was a significant difference in the total score of the psychological well-being with different support utilization level $(P<0.01)$. This indicates that social supports were mutual. On the one hand, the rural left-behind elderly might take part in social activities actively, and support other people, which would obtain the supports from others in turn. On the other hand, the rural left-behind elderly might accept the supports from others, but not refuse others' help.

\section{Acknowledgement}

This research was financially supported by the National Social Science Fund Project of China (14BGL213) and School of Public Management, Guizhou University of Finance and Economics.

\section{References}

[1] J. Hu, B. Wu, X. L. Wu, et al., Analysis of out-patient service utilizations and influence factors among the left-behind elderly in ethnic Miao villages, Chinese journal of public health. 2012, 28 (9) 1176-1178.

[2] M. Nie, Q. H. Wang, Y. S. Yao, Research progress of psychological problems among the left-behind and empty-nest elderly in China, Chinese journal of gerontology. 2011, 31 (12) 2364-2366.

[3] H. Y. Jia, J. Y. Wang, Analysis of mental health status among the rural elderly, Chinese journal of public health. 2007, 23 (6) 763-764.

[4] Z. Y. Chen, Mental health status and psychological intervention among the left-behind elderly in the west national regions, Journal of nursing research. 2008, 22 (12) 3138-3139. 
[5] Z. Y. Wu, S. L. Xu, J. Li, Compilation of psychological well-being questionnaire for the elderly, Chinese journal of clinical psychology. 2002, 10 (1) 1-3.

[6] X. D. Wang, X. L. Wang, H. Ma, A handbook of psychological health assessment scale, revised ed., Chinese mental health magazine, Beijing, 1999:112-117.

[7] L. X. Chen, Y. Yao, Research on the impact of social supports on mental health among the elderly, Journal of population studies. 2005, 29 (4) 73-78.

[8] J. P. Xiong, Mental health and social support condition among the empty-nest elderly in urban areas in Henan, Chinese journal of gerontology. 2011, 31 (11) 2068-2069. 